\title{
Efficacy and Planning in Ophthalmic Surgery - A Vision of Logical Programming
}

\author{
Nuno Maia ${ }^{1}$, Manuel Mariano ${ }^{2}$, Goreti Marreiros ${ }^{3}$, \\ Henrique Vicente ${ }^{4,5}$, and José Neves ${ }^{5(\bowtie)}$ \\ ${ }^{1}$ Departamento de Informática, Universidade do Minho, Braga, Portugal \\ nuno.maia@mundiservicos.pt \\ ${ }^{2}$ Centro Hospitalar Baixo Vouga, EPE, Aveiro, Portugal \\ msmmariano@gmail.com \\ 3 GECAD - Grupo de Engenharia do Conhecimento e Apoio à Decisão, \\ Departamento de Engenharia Informática, \\ Instituto Superior de Engenharia do Porto, Porto, Portugal \\ goreti@dei.isep.ipp.pt \\ ${ }^{4}$ Departamento de Química, Escola de Ciências e Tecnologia, \\ Universidade de Évora, Évora, Portugal \\ hvicente@uevora.pt \\ 5 Centro Algoritmi, Universidade do Minho, Braga, Portugal \\ jneves@di.uminho.pt
}

\begin{abstract}
Different variables should be considered in order to identify the critical aspects that influence ophthalmologic surgery and, in particular, the patient's conditions that can become the key factor in this process, i.e., in situations that can influence the stability and surgery of the patient. Protocol of ophthalmologic surgery has as main concern Glycemic Index, Maximum Blood Pressure, Abnormal Cardiac Index, and Cardiac-Respiratory Insufficiency. Such variables will be used to construct a dynamic virtual world of complex and interacting entities that map real cases of surgical planning situations, understood here as the terms that make the extensions of mathematical logic functions that compete against one another in a rigorous selection regime in which fitness is judged by one criterion alone, its Quality-of-Information. Indeed, one focus is on the development of an Evolutionary Clinical Decision Support System to evaluate patient stability and assist the physicians in the decision of doing or postponing surgery, once cataract is the leading cause of blindness in the world.
\end{abstract}

Keywords: Ophthalmologic surgery $\cdot$ Logic programming $\cdot$ Evolutionary case based reasoning $\cdot$ Knowledge representation and reasoning 